\title{
Distribution of the Crystalline Lens Power In Vivo as a Function of Age
}

\author{
Sien Jongenelen, ${ }^{1}$ Jos J. Rozema,${ }^{1,2}$ and Marie-José Tassignon ${ }^{1}$; on behalf of the EVICR.net and \\ Project Gullstrand Study Group
}

${ }^{1}$ Department of Ophthalmology, University Hospital Antwerp, Edegem, Belgium

${ }^{2}$ Faculty of Medicine and Health Sciences, University of Antwerp, Wilrijk, Belgium

Correspondence: Jos J. Rozema, Department of Ophthalmology, University Hospital Antwerp, Wilrijkstraat 10, 2650 Edegem, Belgium; Jos.Rozema@uza.be.

See the appendix for the members of the Project Gullstrand Study Group.

Submitted: August 26, 2015

Accepted: September 28, 2015

Citation: Jongenelen S, Rozema JJ, Tassignon M-J; on behalf of the EVICR.net and Project Gullstrand Study Group. Distribution of the crystalline lens power in vivo as a function of age. Invest Ophthalmol Vis Sci. 2015;56:7029-7035.

DOI:10.1167/iovs.15-18047
Purpose. To observe the age-related changes in crystalline lens power in vivo in a noncataractous European population.

MetнoDs. Data were obtained though Project Gullstrand, a multicenter population study with data from healthy phakic subjects between 20 and 85 years old. One randomly selected eye per subject was used. Lens power was calculated using the modified Bennett-Rabbetts method, using biometry data from an autorefractometer, Oculus Pentacam, and Haag-Streit Lenstar.

Results. The study included 1069 Caucasian subjects (490 men, 579 women) with a mean age of $44.2 \pm 14.2$ years and mean lens power of $24.96 \pm 2.18$ diopters (D). The average lens power showed a statistically significant decrease as a function of age, with a steeper rate of decrease after the age of 55 . The highest crystalline lens power was found in emmetropic eyes and eyes with a short axial length. The correlation of lens power with different refractive components was statistically significant for axial length $(r=-0.523, P<0.01)$ and anterior chamber depth $(r=-0.161, P<0.01)$, but not for spherical equivalent and corneal power $(P>0.05)$.

Conclusions. This in vivo study showed a monotonous decrease in crystalline lens power with age, with a steeper decline after 55 years. While this finding fundamentally concurs with previous in vivo studies, it is at odds with studies performed on donor eyes that reported lens power increases after the age of 55 .

Keywords: crystalline lens power, age
$\mathrm{T}$ he crystalline lens is a refractive element in the posterior chamber of the eye that has a biconvex shape and a gradient refractive index. ${ }^{1}$ This gradient index originates from a difference in concentration of crystallins located in the cytoplasm of lens fiber cells, with high concentrations in the lens nucleus and lower concentrations in the lens cortex, together with a gradient in distribution of crystallin types. ${ }^{2}$ With age, the human lens grows by a process of epithelial cell division and the formation of differentiated fiber cells that alters the dimensions of the lens, including mass, thickness, radii of curvature, and refractive index. ${ }^{3,4}$ Since the total lenticular power depends on all these parameters, ${ }^{5,6}$ this results in a gradual change in lenticular power as well.

There have been many attempts in the literature to determine the refractive lens power in vitro or in vivo, both of which suffer from their own challenges. In vitro studies, for example, determine the lenticular power using isolated donor lenses that are removed from their natural position and zonular tension. This leads to shape changes equivalent to that of a maximally accommodated lens, ${ }^{7}$ which can be counteracted by using a stretching apparatus to restore the original shape. Furthermore, attention has to be given to storage, avoiding swelling, and handling, which could alter the internal structure of the lens. ${ }^{8}$

In vivo assessment of the lenticular power, on the other hand, is challenging due to the position of the lens inside the eye, which impedes measuring its power directly. There is an indirect method called phakometry, however, that determines the radii of curvature by analyzing the location and relative sizes of the Purkinje reflections. ${ }^{9,10}$ But since these devices are not available commercially, other indirect approaches for estimating lens power have been proposed. One such approach is Bennett's formula, ${ }^{11}$ which calculates the equivalent lens power based on ocular biometry and refraction, assuming that the lens is similar to that of the Gullstrand-Emsley model eye. ${ }^{12}$ This method produces power values similar to those with phakometry ${ }^{13}$ but requires knowledge of lens thickness, which is not always available in clinical practice. In those cases the thin lens formula by Bennett and Rabbetts ${ }^{14}$ can be used instead. The accuracy of these formulas depends on the validity of biometric parameters included, ${ }^{15}$ and they may be modified and refined to match the best prediction of the power of the human lens. ${ }^{16}$

Lens power has an important influence during the emmetropization process in infancy ${ }^{17}$ when the lens power decreases significantly to play a key role in the refractive adjustment of the eye during growth. ${ }^{18,19}$ During this time the crystalline lens loses approximately 20 diopters (D) of power, from 43 to $47 \mathrm{D}$ in infancy to $25 \mathrm{D}$ by the age of $6,{ }^{20}$ which continues to decrease further to 21 to $22 \mathrm{D}$ at the onset of adulthood. ${ }^{21}$ It is much less clear, however, what happens with lens power in middle-aged and older subjects, as there are only 
Table 1. Descriptive Statistics of Each Parameter by Sex and in Total

\begin{tabular}{|c|c|c|c|c|c|}
\hline Variable & $S E, \mathrm{D}$ & $\boldsymbol{K}, \mathbf{D}$ & $A C D, \mathrm{~mm}$ & $L, \mathbf{m m}$ & $\boldsymbol{P}_{L}, \mathbf{D}$ \\
\hline \multicolumn{6}{|c|}{ Males, $N=490$} \\
\hline Mean $\pm \mathrm{SD}$ & $-0.93 \pm 2.24$ & $41.95 \pm 1.37$ & $3.49 \pm 0.39$ & $24.12 \pm 1.10$ & $24.23 \pm 2.10$ \\
\hline Skewness & -0.686 & 0.309 & -0.197 & 0.493 & 0.227 \\
\hline Kurtosis & 1.174 & 0.914 & -0.306 & 0.150 & 1.297 \\
\hline \multicolumn{6}{|c|}{ Females, $N=579$} \\
\hline Mean $\pm \mathrm{SD}$ & $-0.75 \pm 2.09$ & $42.34 \pm 1.40$ & $3.37 \pm 0.38$ & $23.55 \pm 1.05$ & $25.57 \pm 2.06$ \\
\hline Skewness & -0.814 & 0.144 & -0.028 & 0.387 & 0.316 \\
\hline Kurtosis & 2.438 & 0.580 & -0.383 & 0.604 & 0.233 \\
\hline \multicolumn{6}{|l|}{ Total, $N=1069$} \\
\hline Mean $\pm S D$ & $-0.84 \pm 2.16$ & $42.16 \pm 1.40$ & $3.43 \pm 0.39$ & $23.81 \pm 1.11$ & $24.96 \pm 2.18$ \\
\hline Skewness & -0.76 & 0.22 & -0.090 & 0.43 & 0.217 \\
\hline Kurtosis & 1.78 & 0.65 & -0.383 & 0.40 & 0.624 \\
\hline
\end{tabular}

a few epidemiological studies available on this topic. In vitro studies show a linear decrease of the isolated lens power with age, ${ }^{22-24}$ after which some authors report a linear age-related increase after the age of 50 to $60 .^{5,7}$

The purpose of this study was to verify whether this particular relationship between crystalline refractive power and age may also be observed in vivo by studying the agerelated changes in crystalline lens power for a European population, and to examine its relationship with sex and other biometric parameters.

\section{Materials AND Methods}

\section{Subjects}

Subjects were recruited through Project Gullstrand, a European multicenter study with the goal to determine the average biometric values of human eyes. In total, nine centers in six European countries gathered biometry data of healthy, phakic Caucasians in the period between January 2011 and July 2013. Exclusion criteria for the study were subjects younger than 20 years, a refraction outside the interval $\pm 10 \mathrm{D}$, a self-reported history of previous ocular surgery or ocular pathologies, cataract (LOCS III $^{25}$ : nuclear opacities $\leq 2$, other opacity types $<1$ ), amblyopia, wearing hard contact lenses, self-reported systemic diseases (e.g., diabetes, multiple sclerosis), or a pregnancy of more than 5 months. The study adhered to the tenets of the Declaration of Helsinki and received approval from the ethics committee of the Antwerp University Hospital (Ref. B30020072406) and all other participating sites. Signed informed consent was obtained from each volunteer prior to participation.

In total, 1069 individuals (490 males, 579 females) were included, with a mean age of $44.2 \pm 14.2$ years (range, 20-85 years). No cycloplegia was used to remain as close to the everyday physiological condition of the eye as possible. This may have introduced a small refractive shift, especially in the subjects below the age of $50 .^{26}$

\section{Measurements and Calculations}

The objective refraction was measured with an autorefractometer (varies per center), while the anterior chamber depth $(A C D)$ and corneal shape parameters were collected with a Scheimpflug camera (Pentacam, Oculus Optikgeräte, Wetzlar, Germany; Galilei, Ziemer Ophthalmic Systems, Port, Switzerland; Sirius, CSO, Scandicci, Italia). Finally the axial length was determined with a partial coherence biometer (IOL Master, Carl Zeiss, Jena, Germany, or Lenstar, Haag-Streit, Koeniz, Switzerland). At this time the presence of nuclear lens opacification was also checked using the Scheimpflug images. Note that since the biometry was determined with various devices, special statistical considerations are needed to account for between-center differences. ${ }^{27}$

Table 2. Distribution of Biometric Parameters by Age for the Entire Group (Average \pm Standard Deviation)

\begin{tabular}{|c|c|c|c|c|c|c|c|c|c|c|}
\hline Age, $\mathbf{y}$ & $N$ & $S E, \mathrm{D}$ & $L, \mathbf{m m}$ & $\boldsymbol{P}_{L}, \mathbf{D}$ & $\Delta P_{L}, \mathbf{D}$ & \multicolumn{5}{|c|}{ Homogeneous Subsets, SNK } \\
\hline$<25$ & 81 & $-1.66 \pm 1.87$ & $23.92 \pm 1.08$ & $26.17 \pm 2.10$ & & 1 & & & & \\
\hline $25-30$ & 133 & $-1.49 \pm 1.96$ & $23.97 \pm 1.08$ & $25.88 \pm 1.98$ & -0.29 & 1 & 2 & & & \\
\hline $30-35$ & 148 & $-1.37 \pm 2.28$ & $23.89 \pm 1.19$ & $25.76 \pm 2.07$ & -0.12 & 1 & 2 & & & \\
\hline $35-40$ & 110 & $-1.08 \pm 2.05$ & $23.93 \pm 1.07$ & $25.39 \pm 1.84$ & -0.37 & & 2 & 3 & & \\
\hline $40-45$ & 97 & $-0.84 \pm 2.22$ & $23.90 \pm 1.24$ & $25.06 \pm 2.17$ & -0.33 & & 2 & 3 & & \\
\hline $45-50$ & 117 & $-0.81 \pm 1.82$ & $23.70 \pm 0.93$ & $25.17 \pm 1.89$ & 0.11 & & 2 & 3 & & \\
\hline $50-55$ & 107 & $-0.79 \pm 2.15$ & $23.83 \pm 1.21$ & $24.65 \pm 1.68$ & -0.52 & & & 3 & & \\
\hline $55-60$ & 85 & $0.33 \pm 2.04$ & $23.47 \pm 1.08$ & $23.98 \pm 2.10$ & -0.67 & & & & 4 & \\
\hline $60-65$ & 108 & $-0.16 \pm 2.11$ & $23.68 \pm 1.07$ & $23.64 \pm 1.93$ & -0.34 & & & & 4 & \\
\hline $65-70$ & 46 & $0.26 \pm 1.95$ & $23.62 \pm 0.90$ & $23.29 \pm 2.02$ & -0.35 & & & & 4 & \\
\hline$>70$ & 37 & $-0.07 \pm 2.58$ & $23.95 \pm 1.11$ & $22.51 \pm 1.68$ & -0.78 & & & & & 5 \\
\hline
\end{tabular}

$\Delta P_{L}$, difference with previous age category.

SNK, Student-Newman-Keuls post hoc test. 
TABLE 3. Distribution of Lens Power by Refraction

\begin{tabular}{lcccccc}
\hline $\boldsymbol{S E}, \mathbf{D}$ & $<-5$ & $-\mathbf{5}<\boldsymbol{z}<-\mathbf{3}$ & $-\mathbf{3}<\boldsymbol{z}<-\mathbf{1}$ & $-\mathbf{1}<\boldsymbol{z}<+\mathbf{1}$ & $+\mathbf{1}<\boldsymbol{z}+\mathbf{3}$ & $>+3$ \\
\hline$N$ & 47 & 106 & 226 & 538 & 122 & 30 \\
$P_{L}, \mathrm{D}^{*}$ & $24.05 \pm 2.71$ & $24.50 \pm 1.93$ & $25.00 \pm 2.16$ & $25.30 \pm 2.02$ & $24.29 \pm 2.51$ & $24.18 \pm 2.18$ \\
\hline
\end{tabular}

$*$ Mean $\pm \mathrm{SD}$.

As lens thickness was not available for all subjects, lens power $P_{L}$ was calculated using the modified Bennett-Rabbetts equation $^{16}$ :

$$
P_{L}=\frac{L\left(S_{C V}+K_{t o t}\right)-1000 \cdot n}{\left(L-A C D_{c}-c\right)\left(\frac{A C D_{c}+c}{1000 \cdot n}\left(S_{C V}+K_{t o t}\right)-1\right)}
$$

with $L$ the axial length, $S_{C V}=S E /(1-0.014 \cdot S E)$ the spherical equivalent refraction at the corneal vertex, $c=3.304 \mathrm{~mm}$ the average distance between thin lens position and anterior lens surface, and $n=1.336$ the refractive index of aqueous and vitreous humors. ${ }^{16}$ We implemented Manns' correction (Manns F. IOVS 2014;55:ARVO E-Abstract 3785) to account for the influence of the posterior cornea on the lens power calculation by using the total corneal power $K_{t o t}=K_{m, a}+K_{m, p}-K_{m, a} \cdot K_{m, p} \cdot C C T /$ $n_{c}$. Here $K_{m, a}$ and $K_{m, p}$ are the mean anterior and posterior keratometry, respectively; $C C T$ is the central pachymetry; and $n_{c}$ $=1.376$ the corneal refractive index. This also requires defining the $A C D$ as the distance between the second principal plane of the cornea and the anterior lens plane, which is calculated by $A C D_{c}=A C D-\left(C C T \cdot K_{t o t}\right) /\left(1000 \cdot n_{c} \cdot K_{m, a}\right)$.

Although data from both eyes were collected, only one randomly selected eye was used per subject to avoid the influence of high intraindividual correlations of biometric factors.

\section{Statistical Analysis}

This work used biometric data from various sites, which means that minor differences in average age, sex balance, and equipment are to be expected. As this could influence the results, intraclass correlations (ICC) were used to estimate the importance of the between-site differences. If necessary, suitable adjustments could be made.

Each parameter was described by sex and binned per 5 years of age. Student's $t$-test was used to compare the biometric

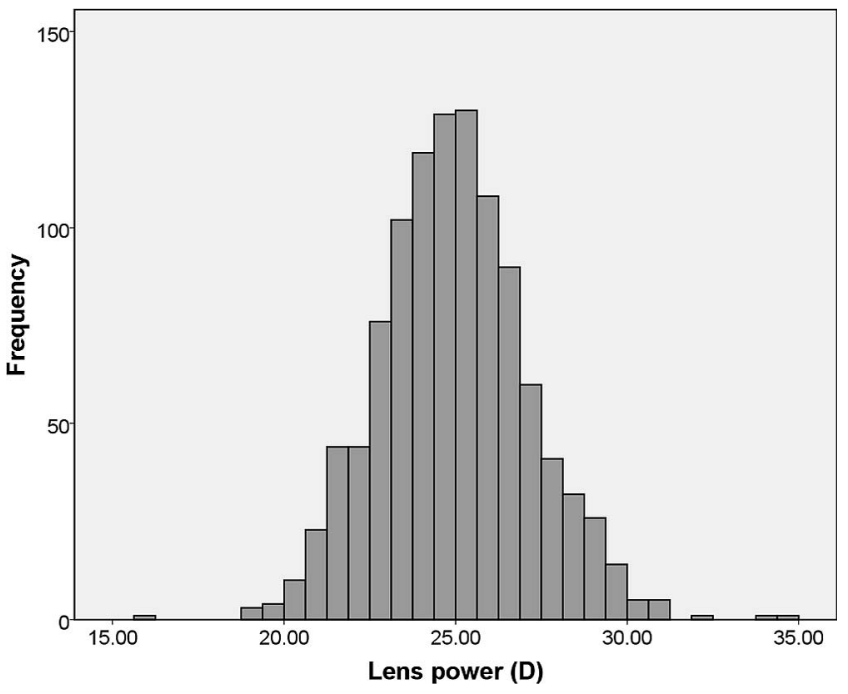

Figure 1. The distribution of refractive lens power for the entire cohort (age range, 20-85 years). parameters by sex. The lens power for each age category was compared through an ANOVA, followed by post hoc testing to assess the progress of lens power with age. Similarly, the influence of axial length and refraction was determined as well. Correlations of the parameters with biometry were analyzed by Pearson correlation coefficients. A univariate general linear model was applied to the data, and a simple main effects analysis evaluated the interaction between age and sex further. Significance levels of 0.05 were used throughout the analysis. The data were analyzed using SPSS version 21.0 for Windows (IBM Corp., Armonk, NY, USA).

\section{Results}

\section{Between-Site Effects}

We used mixed effects models to calculate the ICC for $P_{L}, L$, $A C D, K$, and $S E$ after correction for age and sex differences to estimate systematic between-site effects. These ICC coefficients were found to remain very small (between 0.006 and 0.065), making adjustments for between-site effects unnecessary.

\section{Descriptive Statistics}

Table 1 gives the descriptive statistics for the total population of 1069 Caucasians from six different countries, split up by sex. The mean spherical equivalent refractive power was -0.84 $\pm 2.16 \mathrm{D}$, while the mean refractive power of the lens and cornea were $24.96 \pm 2.18$ and $42.16 \pm 1.40 \mathrm{D}$, respectively. The distribution of the lens power had a broad, Gaussian shape (Fig. 1). Significant differences between male and female eyes were found for corneal power (unpaired $t$-test, $P<0.001$ ), $A C D(P<0.001)$, axial length $(P<0.001)$, and lens power $(P$ $<0.001)$, but not for refraction $(P=0.183)$. Women had shorter eyes, shallower anterior chambers, higher corneal power, and a higher lens power than men.

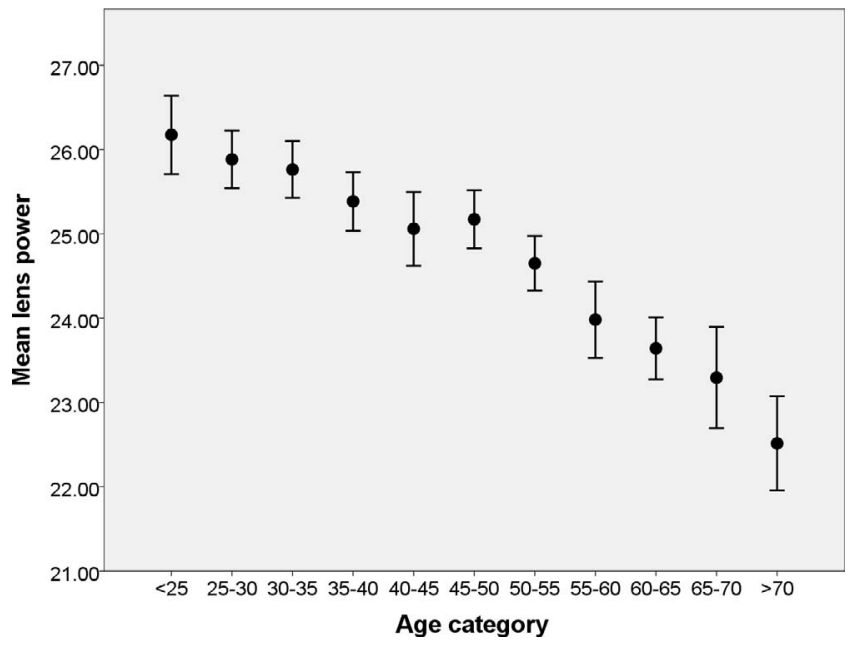

Figure 2. The distribution of refractive lens power per age category. Error bars represent the $95 \%$ confidence interval of the standard error. 

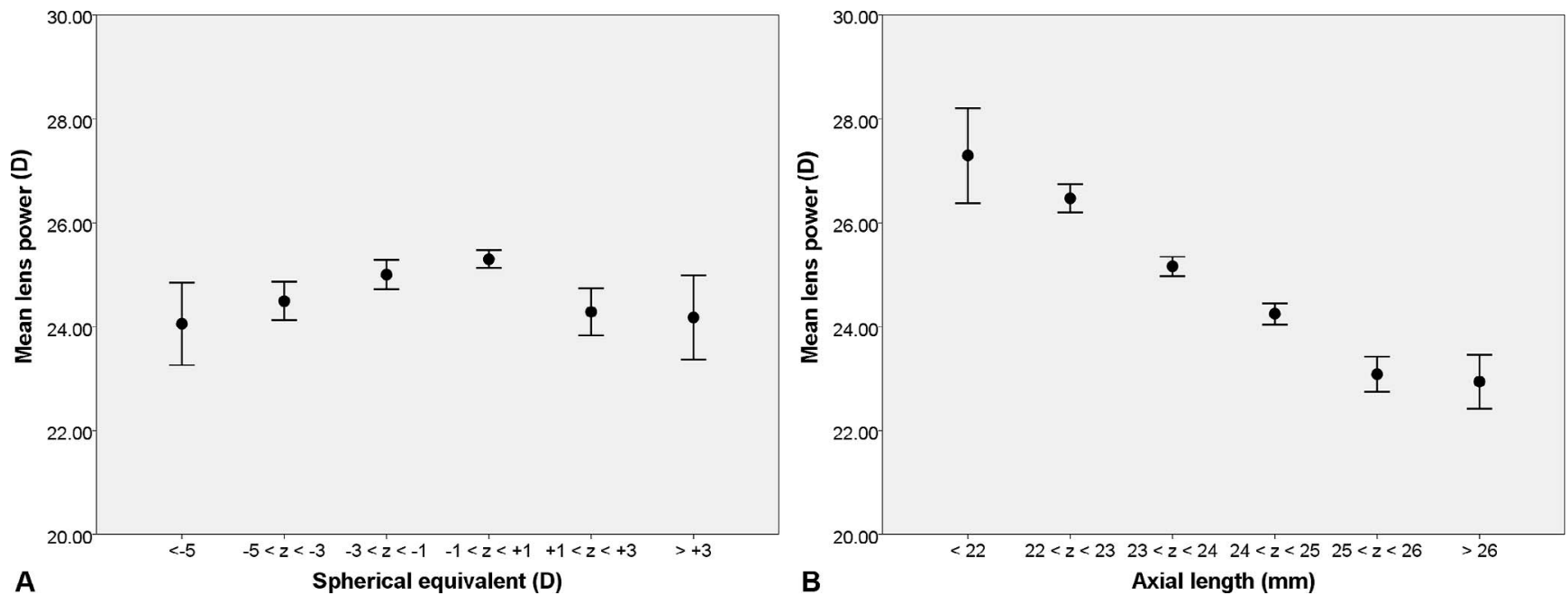

Figure 3. The mean refractive lens power stratified by spherical equivalent (A) or axial length (B). Error bars represent the $95 \%$ confidence interval of the standard error:

\section{Influence of Age}

There was a negative correlation between lens power and age (Pearson $r=-0.437, P<0.01$ ). Binned per 5 years of age, lens power undergoes a near-monotonous decrease (Table 2; Fig. 2), which was found to be significant (ANOVA, $P<0.001$ ). A post hoc Student-Newman-Keuls analysis demonstrated that lens power was more similar for younger age categories, while for older age categories these were less similar (last columns in Table 2). This is indicative of a higher rate of lens power decrease for older ages, which contradicts the in vitro reports.

\section{Correlation With Sex and Biometry}

The correlation of lens power with biometry was significant for axial length $L$ (Pearson $r=-0.523, P<0.01)$ and $A C D(r=$ $-0.161, P<0.01)$, meaning that an increase in lens power is correlated with a decrease in axial length or $A C D$. Spherical equivalent $(P=0.450)$ and corneal power $(P=0.058)$ were not significantly correlated with refractive lens power.

The correlation between refraction and lens power was investigated further by dividing refraction into 2-D bins (Table $3)$. Subsequent ANOVA showed a significant difference between refraction groups $(P<0.001)$, with emmetropic eyes having significantly higher lens powers than either high myopes or hypermetropes (Tamhane's T2 post hoc test). No significant differences were seen between high myopes $(S E<$ $-5 \mathrm{D})$ and hypermetropes $(S E>+1 \mathrm{D}$; Tamhane T2: $P>0.99)$. This is also seen in Figure $3 \mathrm{~A}$, where lens power seems to follow an arc shape as a function of refraction.

Repeating the same analysis by dividing eyes into $1-\mathrm{mm}$ bins according to axial length demonstrated a significant decrease in lens power with increasing axial length (ANOVA, $P<0.001$; Fig. 3B, Table 4).

There was also a small but significant interaction between age and sex for lens power (two-way ANOVA, $P=0.012$ ), which accounted for $27.6 \%$ of the lens power variation. This interaction was analyzed further using a simple main effects analysis, which showed that sex influenced lens power significantly in almost every age category except 60 to 65 ( $F$ $=1.086, P=0.298)$ and $>70(F=2.740, P=0.098)$. A plot of the estimated marginal means of lens power by sex showed nearly parallel lines (Fig. 4), indicating that the changes in lens power occur equally in both sexes.

\section{Discussion}

These results suggest that the in vivo crystalline lens power calculated with the modified Bennett-Rabbetts method follows a biphasic course, with a flat linear decrease at young ages and a faster decrease after the age of 55 . This course is in contrast with the literature, where Glasser and Campbell, ${ }^{5}$ as well as Borja et al., ${ }^{7}$ reported an increase in lens power after the age of 50 to $60,{ }^{5,7}$ Jones et al., ${ }^{23}$ and Atchison et al. ${ }^{28}$ found a monotonous linear decrease, and Olsen et al. ${ }^{29}$ reported no correlation between lens power and age between 55 and 100 years of age. Finally, Iribarren et al. ${ }^{30}$ even reported a very mild increase and subsequent decrease in lens power in Iranian subjects between 40 and 64 years of age. From this overview of the literature we find that, although most of these articles confirm that lens power changes with age and that the rate of this change somehow alters between the age of 50 and 60, in vitro and in vivo studies seem to disagree on the exact course of this altered rate of change (Fig. 5).

The source of this disagreement between studies may lie in the methodology used. In three studies the experiments were performed on postmortem donor material, in which the lenses entered a state of maximal accommodation as they were removed from zonular tension. This can be counteracted by placing the lens in a stretching apparatus, as was done by Glasser and Campbell, ${ }^{5}$ and Borja et al. ${ }^{7}$ Jones et al., ${ }^{23}$ on the other hand, did not stretch their lenses, and consequently reported lens power values that were considerably higher (Fig. 5). While in either case the age-related decrease in isolated lens power was found to match the in vivo decrease in accommodative amplitude quite well, ${ }^{7,23}$ the lens powers and the rates of power change with age reported by the in vitro studies are considerably higher than those of the in vivo studies. This may indicate that even when placed in a stretching apparatus, the optical properties of the lens still differ from the physiological suspension inside the eye.

There are also methodological differences between the four in vivo studies, most prominently in the methods used to determine lens power and cycloplegia of the subjects. Olsen et al. $^{29}$ used a variation of the Olsen IOL formula ${ }^{31}$ on data from noncyclopleged Icelandic subjects between 55 and 100 years old, ${ }^{29}$ while Atchison et al. ${ }^{28}$ performed phakometry on noncyclopleged subjects, Iribarren et al. ${ }^{30}$ applied the Bennett formula to data of middle-aged Iranian subjects after 


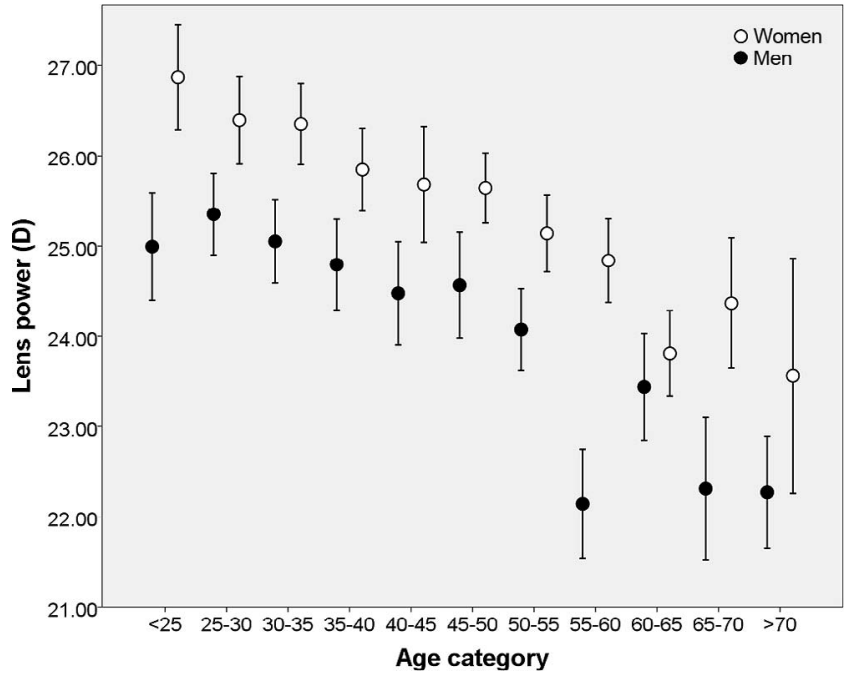

Figure 4. The mean refractive lens power stratified by age category and sex.

cycloplegia, and finally the Bennett-Rabbetts formula applied to noncyclopleged Caucasian subjects aged between 20 and 85 years was used in this study. Given the advanced age of the Olsen et al. subjects and the cycloplegia of the Iribarren et al. data, the influence of accommodation can be excluded from these two data sets. The lack of cycloplegia in the current study may have caused some of our subjects below the age of 50 to accommodate during measurement, which would increase lens power in the younger age categories, thus steepening the lens power decrease on the left-hand side of Figure 2. The influence of this effect can be estimated by referring to a recent article by Morgan et al. ${ }^{26}$ who found that in subjects aged 20 to 50 years the mean difference between cycloplegic and noncycloplegic refraction can be up to 0.20 to $0.25 \mathrm{D}$ for myopes and emmetropes, and up to 0.50 to 0.65 $\mathrm{D}$ for hypermetropes. This corresponds with lens power increases of 0.37 and $0.95 \mathrm{D}$ for myopes/emmetropes and hypermetropes, respectively. Therefore the lens power decrease we found before the age of 50 may have been exaggerated by this amount, although the actual amount is likely to be smaller, as not all subjects will accommodate and the proportion of hypermetropes was less than $20 \%$ in the present cohort.

The lens power formulas used in the in vivo studies are unlikely to be a great source of disagreement either, as the Bennett and the Bennett-Rabbetts formulas have been shown to agree well with each other. ${ }^{16}$ Another issue is the use of the total corneal power (i.e., including the contribution of the posterior corneal surface) rather than the use of the anterior cornea alone. This procedure was first suggested by Manns (Manns F. IOVS 2014;55:ARVO E-Abstract 3785), and can be obtained by either adjusting the keratometric refractive index (e.g., to $n=1.3315$ ) or by combining both the anterior and posterior surface powers into a total power value. This procedure caused our mean lens power value to be approximately $1 \mathrm{D}$ higher in comparison to the literature.

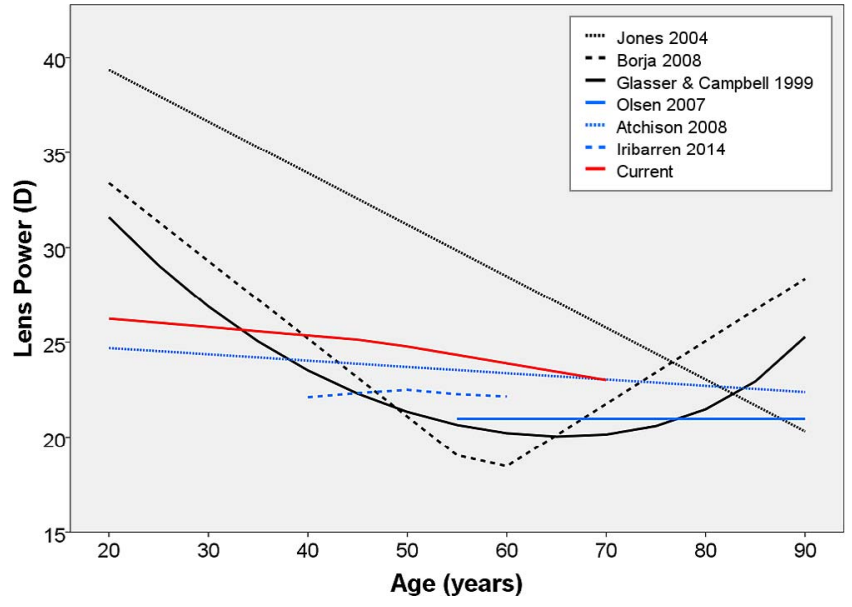

Figure 5. Changes in lens power with age reported in the literature by Glasser and Campbell ${ }^{5}$ (19 eyes, stretched in vitro using ray tracing), Jones et al. ${ }^{23}$ (20 eyes, unstretched in vitro using magnetic resonance imaging), Borja et al. ${ }^{7}$ (51 eyes, stretched in vitro using lens meter), Olsen et al. ${ }^{29}$ (325 eyes, in vivo using Olsen method and noncyclopleged refraction), Atchison et al. ${ }^{28}$ (66 eyes, in vivo using phakometry and noncyclopleged refraction), Iribarren et al. ${ }^{30}$ (1926 eyes, in vivo using Bennett method and cyclopleged refraction), and the current study (1069 eyes, in vivo using Bennett-Rabbetts method and noncyclopleged refraction).

Although the processes behind the gradual decrease in lens power with age are well known, ${ }^{17}$ it remains unclear why the rate of the lens power decrease seems to change between the ages of 50 to 60 years. Glasser and Campbell ${ }^{5}$ and Borja et al. ${ }^{7}$ attributed the increase they found to the presence of cataract in some of their investigated lenses, which is known to cause myopic shifts. ${ }^{32}$ Similarly, Olsen et al. ${ }^{29}$ mentioned that the cataract in some of their subjects may have influenced their lens power values. Finally, in Atchison et al., ${ }^{28}$ Iribarren et al., ${ }^{30}$ and the current study, all subjects with cataractous lenses were excluded, which could be why these studies showed a power decrease after the age of 60 .

Note that the studies above are all cross-sectional rather than longitudinal, which implies that the results may have been influenced by gradual changes within the cohorts used. Examples of such effects are the increase in lens power before the age of 50 seen in Iranian adults, ${ }^{30}$ which was attributed to differences in height, with younger people being taller than older people, or the increasing prevalence of myopia in young subjects, which will undoubtedly influence lens power as well.

According to some studies, refractive error in adults is not significantly associated with crystalline lens power, ${ }^{30,33}$ while others reported a negative correlation, with higher lens powers in myopes. ${ }^{15,29}$ This is in contrast to the positive correlation found in children's eyes, with hypermetropes having higher refractive lens powers than emmetropes or myopes, ${ }^{34,35}$ while the current study suggests that emmetropic eyes have significantly higher lens powers than in high myopes or hypermetropes. The positive correlation between lens power and spherical refraction in myopes also

Table 4. Distribution of Lens Power by Axial Length $(L)$

\begin{tabular}{lcccccc}
\hline $\boldsymbol{L}, \mathbf{m m}$ & $<\mathbf{2 2}$ & $\mathbf{2 2}<\boldsymbol{z}<\mathbf{2 3}$ & $\mathbf{2 3}<\boldsymbol{z}<\mathbf{2 4}$ & $\mathbf{2 4}<\boldsymbol{z}<\mathbf{2 5}$ & $\mathbf{2 5} \boldsymbol{z}<\mathbf{2 6}$ & $>\mathbf{2 6}$ \\
\hline$N$ & 34 & 209 & 401 & 276 & 108 & 41 \\
$P_{L}, \mathrm{D}^{*}$ & $27.30 \pm 2.63$ & $26.47 \pm 2.00$ & $25.16 \pm 1.87$ & $24.25 \pm 1.72$ & $23.08 \pm 1.76$ & $22.94 \pm 1.66$ \\
\hline
\end{tabular}

${ }^{*}$ Mean $\pm \mathrm{SD}$. 
matches the known association between lower lens power and increasing axial length. ${ }^{15,18,29,30,34,36} \mathrm{~A}$ myopic eye is usually longer, thus requiring a weaker lens to emmetropize, while a hypermetropic eye is typically shorter, requiring a stronger lens. At a certain age the correlation between spherical refraction and lens refractive power changes from positive to negative, which Iribarren ${ }^{17}$ explained through the hypermetropic shift that occurs in adulthood. As the prevalence of hypermetropia increases with age ${ }^{37}$ while the lens power decreases, the group of hypermetropes will consist of a mixture of newly developed hypermetropes with low lens power and a small portion of persistent hypermetropes with a higher lens power. ${ }^{17}$ This new balance could reverse the correlation between lens power and refraction, and would explain why in the present study lens power is lower in the hypermetropic group in comparison with the emmetropic group. Still, our study shows higher lens powers in emmetropic subjects than in myopic subjects, similar to the correlation found in childhood. This could be explained by the fact that cataractous lenses were not included in this study, which would have increased lens power in older age due to a cataract-induced myopic shift. Through exclusion of cataractous lenses, the myopic subpopulation consisted largely of myopes since childhood, thus having a lower lens power. This is in contrast with the Reykjavik Eye Study ${ }^{29}$ and the CIEMS study, ${ }^{15}$ where lens power calculations were based on adults over the age of 50 and where cataractous lenses were not excluded.

In order to shed some light on these issues, we recommend that future population-based studies follow a longitudinal setup to further explore the balance between refraction, lens power, and ocular biometry.

\section{Acknowledgments}

Project Gullstrand, of which this work is a part, is supported by a grant by the Flemish government agency for Innovation by Science and Technology (IWT 110684).

Disclosure: S. Jongenelen, None; J.J. Rozema, None; M.-J. Tassignon, None

\section{References}

1. American Academy of Ophthalmology. Fundamentals and Principles of Opthalmology. San Francisco, CA; 1991:63-65.

2. Zhao H, Magone MT, Schuck P. The role of macromolecular crowding in the evolution of lens crystallins with high molecular refractive index. Phys Biol. 2011;8:046004.

3. Bron A, Vrensen G, Koretz J, Maraini G, Harding J. The ageing lens. Ophthalmologica. 2000;214:86-104.

4. Brown NP, Koretz JF, Bron AJ. The development and maintenance of emmetropia. Eye. 1999;13:83-92.

5. Glasser A, Campbell MC. Biometric, optical and physical changes in the isolated human crystalline lens with age in relation to presbyopia. Vision Res. 1999;39:1991-2015.

6. Alió JL, Schimchak P, Negri HP, Montés-Micó R. Crystalline lens optical dysfunction through aging. Ophthalmology. 2005;112: 2022-2029

7. Borja D, Manns F, Ho A, et al. Optical power of the isolated human crystalline lens. Invest Ophthalmol Vis Sci. 2008;49: 2541.

8. Augusteyn RC, Rosen AM, Borja D, Ziebarth NM, Parel J-M. Biometry of primate lenses during immersion in preservation media. Mol Vis. 2006;12:740-747.
9. Dunne M. Scheme for the calculation of ocular components in a four-surfaced eye without need for measurement of the anterior crystalline lens surface Purkinje images. Ophthalmic Physiol Optics. 1992;12:370-375.

10. Garner LF. Calculation of the radii of curvature of the crystalline lens surfaces. Ophthalmic Physiol Optics. 1997; 17:75-80.

11. Bennett A. A method of determining the equivalent powers of the eye and its crystalline lens without resort to phakometry. Ophthalmic Physiol Optics. 1988;8:53-59.

12. Emsley H. Visual Optics. 5th ed., Vol. 1. London: Butterworth; 1952:40-42, 344, 360-361.

13. Dunne M, Barnes D, Royston J. An evaluation of Bennett's method for determining the equivalent powers of the eye and its crystalline lens without resort to phakometry. Ophthalmic Physiol Optics. 1989;9:69-71.

14. Rabbetts RB. Bennett \& Rabbett's Clinical Visual Optics. 4th ed. Butterworth-Heinemann: London: 2007:223-227, 412$413,425-426$.

15. Iribarren R, Morgan IG, Nangia V, Jonas JB. Crystalline lens power and refractive error. Invest Ophthalmol Vis Sci. 2012; 53:543-550.

16. Rozema JJ, Atchison DA, Tassignon M. Comparing methods to estimate the human lens power. Invest Ophthalmol Vis Sci. 2011;52:7937-7942.

17. Iribarren R. Crystalline lens and refractive development. Prog Retin Eye Res. 2015;47:86-106.

18. Mutti DO, Mitchell GL, Jones LA, et al. Axial growth and changes in lenticular and corneal power during emmetropization in infants. Invest Ophthalmol Vis Sci. 2006;10:188-189.

19. Garner L, Yap M, Scott R. Crystalline lens power in myopia. Optom Vis Sci. 1992;69:863-865.

20. Wood IC, Mutti DO, Zadnik K. Crystalline lens parameters in infancy. Ophthalmic Physiol Optics. 1996;16:310-317.

21. Koretz JF, Cook CA. Aging of the optics of the human eye: lens refraction models and principal plane locations. Optom Vis Sci. 2001;78:396-404.

22. Moffat BA, Atchison DA, Pope JM. Age-related changes in refractive index distribution and power of the human lens as measured by magnetic resonance micro-imaging in vitro. Vision Res. 2002;42:1683-1693.

23. Jones CE, Atchison DA, Meder R, Pope JM. Refractive index distribution and optical properties of the isolated human lens measured using magnetic resonance imaging (MRI). Vision Res. 2005;45:2352-2366.

24. Glasser A, Campbell MC. Presbyopia and the optical changes in the human crystalline lens with age. Vision Res. 1998;38: 209-229.

25. Chylack LT, Wolfe JK, Singer DM, et al. The lens opacities classification system III. Arch Ophthalmol. 1993;111:831-836.

26. Morgan IG, Iribarren R, Fotouhi A, Grzybowski A. Cycloplegic refraction is the gold standard for epidemiological studies. Acta Ophthalmol. In press.

27. Rozema JJ, Wouters K, Mathysen DG, Tassignon M-J. Overview of the repeatability, reproducibility, and agreement of the biometry values provided by various ophthalmic devices. $\mathrm{AmJ}$ Ophthalmol. 2014;158:1111-1120, e1.

28. Atchison DA, Markwell EL, Kasthurirangan S, Pope JM, Smith G, Swann PG. Age-related changes in optical and biometric characteristics of emmetropic eyes. $J$ Vis. 2008;8:29. 
29. Olsen T, Arnarsson A, Sasaki H, Sasaki K, Jonasson F. On the ocular refractive components: the Reykjavik Eye Study. Acta Ophthalmol Scand. 2007;85:361-366.

30. Iribarren R, Morgan IG, Hashemi $\mathrm{H}$, et al. Lens power in a population-based cross-sectional sample of adults aged 40 to 64 years in the Shahroud Eye Study. Invest Ophthalmol Vis Sci. 2014;55:1031-1039.

31. Olsen T. The Olsen formula. In: Shammas HJ, ed. Intraocular Lens Power Calculations. Thorofare, NJ: Slack, Inc.; 2004;2740.

32. Samarawickrama C, Wang JJ, Burlutsky G, Tan AG, Mitchell P. Nuclear cataract and myopic shift in refraction. Am J Ophthalmol. 2007;144:457-459.

33. Stenstrom $S$. Investigation of the variation and the correlation of the optical elements of human eyes. Am J Optom Arch Am Acad Optom. 1948;25:1-71.

34. Iribarren R, Morgan IG, Chan YH, Lin X, Saw S-M. Changes in lens power in Singapore Chinese children during refractive development. Invest Ophthalmol Vis Sci. 2012;53:5124-5130.

35. Jones LA, Mitchell GL, Mutti DO, Hayes JR, Moeschberger ML, Zadnik K. Comparison of ocular component growth curves among refractive error groups in children. Invest Ophthalmol Vis Sci. 2005;46:2317-2327.

36. Tomomatsu T, Kono S, Arimura S, et al. Relationship between lenticular power and refractive error in children with hyperopia. Clin Ophthalmol. 2013;7:601.

37. Hashemi H, Iribarren R, Morgan IG, Khabazkhoob M, Mohammad K, Fotouhi A. Increased hyperopia with ageing based on cycloplegic refractions in adults: the Tehran Eye Study. Br J Ophthalmol. 2010;94:20-23.

\section{APPENDiX}

\section{Project Gullstrand Study Group}

Study sponsor: EVICR.net-European Vision Institute Clinical Research Network, AIBILI, Azinhaga de Santa Comba, Celas, 3000-548 Coimbra, Portugal

Coordinating investigator: Jos J. Rozema

Coordinating center: EVICR.net

Data collection by EVICR.net clinical sites (CS):

- CS 02-Mainz (Germany): Katrin Lorenz, Mascha von Trentini

- CS 07-Alicante (Spain): Espranza Sala Pomares, Maria José Garcia Corral, Laurent Bataille, Jorge L. Alió

- CS 12-Antwerp (Belgium): Jos J. Rozema, Sien Jongenelen, Irene Ruiz-Hidalgo, Nadia Zakaria, Sorcha Ní Dhubhghaill, Carina Koppen, Marie-José Tassignon

- CS 27-Leipzig (Germany): Franziska Georgia Rauscher, Maria Teresa Blüsch, Jens Dawczynski, Peter Wiedemann

- CS 36-Rome (Italy): Luigi Mosca, Laura Guccione, Monica Riso, Maria Emanuela Toro, Alessandra Rosati

- CS 38-Barcelona (Spain): Francesco Duch, Raimon Escude, Alexia Martinez, Antonio Morilla-Grasa

- CS 51-Valencia (Spain): Cristina Peris-Martínez, Amparo Díez Ajenjo, Carmen Garcia Domene

- CS 51-Girona (Spain): Maria Bozal De Febrer, Teresa Torrent Solans, Alicia Verdugo Gazdik

- CS 60-Tel Aviv (Israel): David Varssano, Barbara Gold

- CS 63-Chieti (Italy): Lisa Toto, Alessandra Mastropasqua, Leonardo Mastropasqua

- CS 67-Milan (Italy): Francesco Fasce, Alessandra Spine1li, Karl Knutsson, Giovanni Fogliato 